\title{
Computing and Combinatorics
}

\author{
Zhipeng Cai ${ }^{1} \cdot$ Alexander Zelikovsky $^{2}$
}

Received: 17 May 2016 / Accepted: 8 June 2016 / Published online: 11 July 2016

(C) Springer Science+Business Media New York 2016

This special issue of Algorithmica consists of four selected papers that were presented at the 20th International Computing and Combinatorics Conference (COCOON 2014), held in the city of Atlanta, Georgia, USA, during August, 2014. The papers have been expanded and rigorously reviewed according to journal standards. COCOON 2014 provided an outstanding forum for researchers to exchange ideas and share experiences in the area of theoretical computer science and combinatorics.

The paper "Approximating maximum agreement forest on multiple binary trees" by Jianer Chen, Feng Shi, and Jianxin Wang studies the Maximum Agreement Forest (MAF) problem on multiple binary phylogenetic trees. The MAF problem seeks a largest common subforest of a collection of phylogenetic trees on the same leaf labelset.

The paper "Building above read-once polynomials: identity testing and hardness of representation" by Meena Mahajan, B. V. Raghavendra Rao, and Karteek Sreenivasaiah investigates further structural properties of Read-Once Polynomials (ROPs) and polynomials that can be expressed as polynomial functions of a small number of ROPs. Their observations result in efficient algorithms on special classes of bounded-read formulas.

The paper "Diffuse reflection radius in a simple polygon" by Eli Fox-Epstein, Csaba Tóth, and Andrew Winslow derives the best possible bound of the number of diffuse reflections for every simple polygon in general position with $n$ walls to be illuminated from a single point light source. The authors also show that the minimum number of

\footnotetext{
$凶$ Zhipeng Cai

zcai@gsu.edu

1 Georgia State University, 25 Park Place, Suite 741, Atlanta, GA 30303, USA

2 Georgia State University, 25 Park Place, Suite 749, Atlanta, GA 30303, USA
} 
diffuse reflections to illuminate a given simple polygon from a single point can be approximated up to an additive constant in polynomial time.

The paper "On edge-unfolding one-layer lattice polyhedra with cubic holes" by Meng-Huan Liou, Sheung-Hung Poon, and Yu-Jie Wei focuses on the edge-unfolding problem on several classes of one-layer lattice polyhedra with cubic holes. It is guaranteed that no self-overlapping can occur in the flattened patch.

We would like to thank the authors for contributing their works, and program committee members and external reviewers for volunteering their time to review the papers. We also thank Dr. Ming Yang Kao, the Editor-in-Chief of Algorithmica for providing the opportunity to edit this special issue.

\section{Guest Editors}

